\title{
DE UMA DISPOSIÇÃO INSOLITA DAS VEIAS POPLITEAS E FEMURAFS
}

Nota de LUIZ SPLENDORE e IOÃO BAPTISTA DE BERNARDES LIMA (terceirannistas)

Damos aqui a descripção de um achado obtido, durante os exercicios escolares de dissecção deste anno, nos membros inferiores de um individuo adulto, preto e referente ao comportamento não habitual das vv. profundas da região poplitéa e, correlativamente, cias vv. profundas da região anterior da coxa.

A nossa attenção foi levada á constatação das disposições anormaes quando nos occupavamos da dissecção do n. sciatico do lado esquerdo. Completamos, após o estudo minucioso deste membro, a dissecção, já iniciada por outros dois collegas, do membro direito sendo que nois foi possivel verificar bilateralmente, com poucas discordancias, o mesmo comportamento.

As disposições insolitas das veias que vamos descrever não são novas e tão pouco extremamente raras, sendo sua interpretação relativamente facil. Apezar disso, achamos opportuno notar, ainda uma vez, a sua occurrencia, com uma descripção minuciosa, levando em conta o seu interesse cirurgico.

A' direita, no espaço intergemellar, isto é, no triangulo inferior do losango popliteo, em lugar de encontrarmos um tronco venoso unico. satellite da a. poplitea, considerado como constante pela maioria dos AA. achamos, ao contrario, dois troncos vencios que acompanham a arteria homonyma, dispusição esta que, por sua vez, é considerada como normal por outros AA. (ROBINEAU), e isso devido a falta de reunião das veias profundas da perna.

Na parte proximal da região poplitéa, isto é, na porção correspondente ao planum popliteum do femur, dos dois troncos venosos satellites da a. ppoplitea, existentes na porção distal do mesmo cavo, um sómente se conserva tal; entretanto existe um terceiro tronco venoso, que decorre tambem como "v. comitans" da a. poplitea até o canal de Hunter e mais além. Desse modo, emquanto que na porção distal temos dois troncos venosos, na porção proximal, em relação mais ou menos immediata com a a. poplitea, existem tres delles.

Os dois troncos venosos satellites do segmento intergemellar da a. poplitea são distinctos desde sua origem, têm com a arteria homonyma re- 
lações proprias e se comportam de modo diverso na sua continuação proximal, como foi porsivel demonstrar com uma dissecção cuidadosa, mesmo ifaltando uma injecção artificial.

Um desses troncos, o menor, ao nivel do arco do solear, é lateral á a. poplitéa e situado no mesmo plano: resulta claramente da confluencia de algumas veias dos mm. solear, gemeo lateral, popliteo, da parte alta dos $\mathrm{mm}$. profundos da região posterior da perna e das duas veias satellites da a. tibial anterior, que levam ao tronco a contribuição maior. O tronco assim constituido e que podemos considerar $\mathbf{v}$ poplitéa lateral, dirige-se. mantendo as relações ditas com a arteria, para cima e recebe as vv. articulares inferiores e superiores lateraes: logo ao nivel das inserções condyloideanas mais altas do m. gemeo lateral, recebe tambem uma grossa veia, de percurso transversal de dentro para fóra, que representa a continuação parcial, ventralmente á a. poplitéa, das vv. articulares medias e das superiores mediaes. Estas, no seu conjuncto, teem uma disposição plexiforme; um dos ramos deste plexo abre-se, como dissemos, na v. poplitéa lateral; um outro ramo, mais delgado, com percurso sagital, vae-se abrir no grosso tronco venoso poplitéo postero-medial, do qual fallaremos mais adiante; um terceiro ramo, mais calibroso e de percurso ascendente, acompanha o lado medial da a. poplitéa no plano popliteo, como veremos daqui a pouco. Deste modo, por causa das communicações deste systema plexiforme, que corresponde ás vv. articulares nedias e superiores mediaes, com os dois troncos venosos popliteos, a a. poplitéa fica como que abraçada por um laço venoso volumoso, cuja concavidade olha para traz. A veia então, que até este ponto decorria ao lado externo da a. poplitea, se faz um pouco posterior e nesta sua posição latero-dorsal acompanha a arteria até a abertura inferior do canal de Hunter.

A a. poplitea, no seu percurso, ao nivel do plano popliteo, além desta veia comitante postero-lateral, cujo calibre se póde avaliar em cerca de 4 mms., é ainda acompanhada por uma outra que decorre ao lado medial da arteria e da qual, como o tronco popliteo lateral, condivide a direç̧ão obliчua para cima e para dentro. Esta veia comitante medial representa, essencialmente, uma das continuações das vv. articulares com disposição plexiforme, antes descripta.

Os dois troncos venosos satellites da a. poplitea apresentam no seu percurso, ao nivel do plano popliteo, de distancia em distancia, tres anastomoses obliquas, que se dirigem do tronco medial para o lateral e de baixo para cima e abraçam o contorno posterior da a. poplitea. Estas duas vv. comitantes da porção proximal da a. poplitea são distinguiveis na sua reciproca posição tambem ao longo do canal de Hunter; neste percurso mesmo existe uma grossa anastomose, ventral á arteria, entre os dois troncos venosos.

No ponto em que se faz evidente a união aponevrotica do tendão do $\mathrm{m}$. grande adductor com a aponevrose do $\mathrm{m}$. vasto medial para constituir a pa- 
rede ventral do canal de Hunter, a $\mathbf{v}$ medial se faz posterior e vae se lançar na continuação da veia que antes é postero-lateral. O tronco venoso resultante dessa anastomcse tem um calibre egual ao da a. femural; na sua origem acha-se situada postero e medialmente á arteria, á qual é fortemente unida pela habitual bainha commum; recebe aqui e alli pequenas vv. musculares e se continua nesta posição até á parte alta do triangulo de Scarpa, onde conflue com a continuação do tronco venoso muito mais calibroso, que vamos descrever.

Este tronco venoso que resulta, ao nivel do annel do solear, da continuaऽão das vv. tibio-peroneiras, occupa, na porção distal do losango popliteo, uma posição ao mesmo tempo dorsal e medial á a. poplitea e a recobre portanto parcialmente atraz; por sua vez é recoberta pelo m. gemeo medial e ciecorre ao lado do n. tibial posterior. Desde sua orígem, esta v. poplitéa postero-medial apresenta um calibre duplo, pelo menos, da veia decorrente lateralmente: recebe no seu percurso as vv. articulares inferiores mediaes, vv. gemeas e pelo seu contorno anterior uma larga contribuição das vv. articulares supero-mediaes, já lembrada; recebe tambem, $2 \mathrm{cms}$. acima, no seu contorno postero-lateral, a v. saphena externa. Ella, até o ponto em que recebe a contribuição das vv. articulares superiores mediaes, é, como dissemos, postero-medial á a. poplitêa: mais para cima do espaço intercondyloideo, se dirige verticalmente para o alto, como a bissectriz do triangulo popliteo superior e porisso cruza muito obliquamente, de dentro para fóra, a a. poplitéa; esta, na sua sahida pelo canal de Hunter, sendo acompanhada pelas duas vv. comitantes descriptas, é claramente medial e ventral ao tronco venoso em questão; este, por sua vez, é acompanhado lateralmente pelo n. tibial posterior, sendo que o n. peroneiro é ainda mais lateral ao n. tibial. Temos que notar que a divisão do n. sciatico é, analogamente á outros casos da assim chamada "duplicidade da v. poplitéa muito alta, verificando-se ao nivel do bordo superior do $\mathrm{m}$. quadrado crural. Ao grosso tron๖o venoso, continuação das vv. tibio-peroneiras, não se pode dar uma denominação topographica exacta, porquanto si é postero-medial á arteria no triangulo popliteo inferior, vem a ser postero-lateral no triangulo superior.

Na parte media do cavo popliteo, esta veia principal é fortemente ligada á a. poplitéa e ás vv. satellites, como de habito; mais para cima, se afasta do feixe artero-venoso; apresenta um calibre de $8 \mathrm{mms}$. mais ou menos, recebe larga contribuição das veias procedentes dos $\mathrm{mm}$. posteriores da coxa, particularmente dos $\mathrm{mm}$. semimembranoso e semitendinoso, pelos quaes é completamente recoberta para traz, sendo, por sua vez, collocada no angulo diedro delimitado pela curta porção do $\mathrm{m}$. biceps, lateralmənte, e pelo corpo muscular da porção ischio-femural do $\mathrm{m}$. grande adductor, medialmente. Para cima afasta-se gradativamente do n. tibial posterior e é recoberta, lateralmente, pela longa porção do $\mathrm{m}$. biceps, repousando sobre a superficie dorsal do m. vasto lateral 
Finalmente, a cerca de $4 \mathrm{cms}$. proximalmente ao contorno superior da abertura dorsal do canal de Hunter, passa da região dorsal da coxa para a região ventral, acompanhando, com percurso inverso, a 3.a a. perfurante i. é, o ramo terminal da a. femural profunda, decorrendo entre os feixes medios do $\mathrm{m}$. grande adductor e cruzando o bordo inferior do $\mathrm{m}$. pequeno adductor: aliás, a a. f. profunda tem os ramos typicos, sendo as duas vrimeiras aa. perfurantes, relativamente calibrosas, originadas isoladamente do tronco da arteria e com percurso habitual.

Assim, a grossa veia, que decorre dorsalmente na parte inferior da região posterior da cuxa, é, em grande parte, independente do percurso das aa. poplitea antes e femural propria, depois. Deve-se accrescentar que, pouco antes de se tornar ventral, envia para cima um ramo que se continua com uma das veias que acompanham a $2 \mathrm{a}^{\mathrm{a}}$ a perfurante.

$\mathrm{Na}$ região do triangulo de Scarpa, acha-se profundamente situada no angulo diedro delimitado pelos $\mathrm{mm}$. medio adductor medialmente, vasto medial lateralmente, seguindo assim o tronco da a. f. profunda á qual póde-se depois considerar como satellite.

No seu percurso ulterior approxima-se gradativamente da a. femural e finalmente conflue, ao nivel da origem da a. f. profunda, cerca de $5 \mathrm{cms}$. càa arcada femural, com a veia comitante da a. femural do canal de Hunter para cima: esta v. satellite da a. femural, pelo calibre muito menor, parece deste modo um ramo collateral confluente na v. f. profunda, que representa a continuação da v. dorsal principal da região poplitéa e depois da região media da coxa.

O tronco venoso unico, resultante da fusão da v. comitante, relativamente pequena, da v. femural e da grossa veia que decorre junto á a. profunda, isto é, a v. femural propriamente dita, adquire depois as relações ordinarias com a arteria; isto é, se colloca medialmente á arteria e, pelo seu calibre de $1 \mathrm{~cm}$. mais ou menos de diametro, a recobre parcialmente para diante, sendo os dois vasos unidos intimamente: temos que lembrar que na v. femural, $2 \mathrm{cms}$. acima da confluencia dos seus dois ramos constituintes, como de habito, abre-se a v. saphena magna.

A' esquerda, apresenta-se, fundamentalmente, a mesma disposição já descripta á direita, porém, com algumas differenças que vamos resumir.

No segmento distal da região poplitea, ou mais propriamente a cm. 1,5, approximadamente, abaixo do annel do solear, se constitue, da confluencia de um grosso tronco tibio-peroneiro com o tronco commum das vv. tibiaes anteriores, uma unica v. poplitéa.

Esta veia, de calibre mais ou menos de $7 \mathrm{mms}$., de paredes espessas, ఓortemente ligada, como de norma, á porção correspondente da a. poplitea, $\in \mathrm{m}$ relação á qual é postero-medial, é cruzada, muito obliquamente, na sua 
superficie posterior e de fóra para dentro, pelo n. tibial posterior, que a acompanha no seu percurso, sendo esta relação com a veia, immediata; é cruzada mais em baixo, tambem de fóra para dentro, pelo corpo muscular do $\mathrm{m}$. plantar delgado.

$\mathrm{Na}$ parte media do cavo poplitéo, este tronco venoso recebe lateralmente as vv. gemeas lateraes; pouco acima da interlinha articular, envia, medialmente um ramusculo muito pequeno, de cerca de $2 \mathrm{mms}$. de calibre, que se dirige para cima e para dentro e, após um percurso de $1 \mathrm{~cm}$. maís ou menos, se une ás vv. gemeas mediaes num unico tronco, que conflue, logo, com o mesmo grosso tronco venoso popliteo, delimitando assim uma lapella fissural longitudinal, de $2 \mathrm{cms}$. de comprimento. Ao nivel de um plano tangente ás faces superiores dos condylos femuraeis, abrem-se, na parede anterior do grande tronco venoso, e isoladamente, as vv. articulares medias e superiores mediaes e lateraes, tambem com disposição plexiforme; estas, mergulhadas no tecido adiposo da região, abraçam por isso medial e lateral mente a a. poplitéa antes de se abrir na veia homonyma. A contribuição destas vv. articulares medias e superiores determina, ao nivel da parte superior dos condylos femuraes, um brusco augmento de calibre do tronco venoso popliteo, que, mesmo estando vasio, pode-se avaliar em $12 \mathrm{mms}$ : esta porção tem o aspecto e o significado de um verdadeiro confluente ou bulbo venoso.

Do contorno antero-medial deste bulbo venoso, logo acima da abertura das vv. gemeas mediaes, origina-se com duas raizes, depois logo confluentes a formar um tronco unico e porisso delimitando uma lapella ovoidal (cerca de cm. 1,5), um tronco venoso que acompanha, antes indiviso, depois com disposição plexiforme, a porção proximal da a. poplitéa, occupanco na sua parte indivisa uma posição medio-dorsal relativamente á arteria. Um outro tronco venoso, de calibre irregular, quasi como varicoso, se origina tambem lateralmente á a. poplitéa com duas raizes: uma do contorno antero-lateral do grande confluente venosio, outra que apparece em continuação com a formação plexiforme das vv. articulares superiores; este ultimo vae se collocar lateralmente á porção proximal da a. poplitéa.

O confluente venoso popliteo se resolve, assim, em um grosso tronco venoso longitudinal, que percorre verticalmente a porção superior do losango popliteo e se conserva dorsal ao femur até a parte superior da região posterior da coxa, e num grupo de vv. longitudinaes com disposição claramente plexiforme, comitantes dá a. poplitéa até o canal de Hunter e além.

De facto, as duas veias, medial e lateral, comitantes da porção proximal cia arteria, originadas do confluente venoso popliteo, se dividem, aqui e alli, no seu percurso, anastomosando-se entre si por diversos ramos com direcção prevalentemente longitudinal, de modo que a dissecção revela que, em alguns pontos, a a. poplitéa opparece costeada por quatro ou cinco ra- 
mos venosos delimitando lapellas, particularmente evidentes em relação á sua superficie dorsal.

A disposição plexiforme das vv. comitantes da porção proximal da a. poplitéa é muito mais accentuada á esquerda que á direita, onde vimos dois troncos venosos mais calibrosos, unidos entre si com anastomoses obliquas. Por isso, mais do que de vv. comitantes da a. poplitea, á esquerda poder-se-

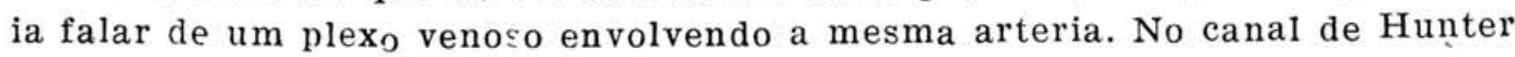
existe a reducção deste plexo em do:s troncos venosos de calibre muito desigual. Uma veia principal acompanha a a. femural em posição posterior e um pouco lateral até á parte superior do triangulo de Scarpa $(5 \mathrm{cms}$. abaixo da arcada crural): esta veia, em todo o seu percurso tem um calibre inferior (4 $\mathrm{mms}$ ) ao da a. femural e porisso esta arteria a recobre completamente para diante. Uma outra veia tem um calibre de mms. 1,5-2 e corre medialmente a a. femural do canal de Hunter para cima; as duas vv. comitantes da a. femural recebem aqui e alli pequenas vv. musculares e são mesmo, particularmente atraz da arteria, unidas por finas porém raras anastomoses.

O tronco venoso lateral, satellite da a. femural na parte superior do triangulo de Scarpa, se faz nitidamente posterior á arteria; une-se, sempre atraz da arteria, com o pequeno tronco medial acerca de $6 \mathrm{cms}$. da arcada crural, no mesmo ponto, pois, em que conflue com o grosso tronco venoso dorsal.

A continuação vertical do confluente venoso popliteo corresponde no seu percurso e nas suas relações, exactamente a quanto descrevemos sobre a mesma formação no lado direito: sómente apparece mais calibroso que o direito e isso devido, sem duvida, ao facto que as vv. satellites do segmento proximal da a. poplitéa direita representam pelo seu calibre, uma via cie defluxo mais larga que o plexo venoso do tracto correspondente do lado esquerdo. J e outra parte a $\mathrm{v}$. poplitéa dorsal, que ao nival de sua origem recobre completamente por cistraz a a. poplitéa, se afasta desta, seja no sentido antero-posterior, seja no transversal; recebe no seu contorno posterior a v. saphena parva um pouco mais acima que a direita; mais proximalmente envia um ramo notavel que se faz satellite da 3.a a. perfurante: de resto, tem com os nn. tibial posterior e peroneiro, aqui tambem prematuramente separados, e com os musculos, as mesmas relações já antes descriptas.

Emfim, este grosso tronco venoso posterior, que decorre acompanhado pelo n. tibial posterior na região posterior da coxa, passa, como á direita, para a região ventral, medialmente ao femur. seguindo o percurso da 2.a a. perfurante da femural profunda, a qual é relativamente mais calibrosa que habitualmente, representando antes o ramo principal da a. f. profunda. No que se refere a esta arteria, devemos accrescentar que a 1.a a. perfurante nasce de um tronco commum com a a. circumflexa medial do femur, separando-se desta depois de 2 cms. de percurso; a 3.a a. perfurante, muito delgada, comporta-se no seu trajecto, como habitualmente. A grossa 
veia dorsal, antes de atravessar os feixes medios do $\mathrm{m}$. grande adductor, envia ainda um grosso tronco, que continúa o decurso ascendente, unindo-se depois ás vv. plexiformes satellites do tronco commum da 1.a perfurante e a. circumflexa femuris medialis.

$\mathrm{Na}$ região do triangulo de Scarpa, a veia que vem da região dorsal costeando os feixes medios do $\mathrm{m}$. grande adductor e o bordo inferior do $\mathrm{m}$. pequeno adductor, segue depcis o tronco da a. f. profunda, apresentando porém um calibre que é pelo menos o quadruplo da mesma arteria; colloca-se desde o começo medialmente á ella e, conflunido com o tronco das pequenas vv. comitantes da a. femural, que, como a direita e mais que a direita, podem ser consideradas como seus affluentes collateraes, vae constituir a v. femural propriamente dita: emfim, esta decorre medialmente á arteria, como de habito. $E^{\prime}$ de notar que o tronco commum da a. circumflexa medial do femur da 1.a a. perfurante passa numa botoneira delimitada precisamente pela v. satellite da a. f. profunda, sendo os dois ramos que constituem a lapella, equipolentes; o dito tronco arterioso é mesmo acompanhado superiormente e para traz por um grosso tronco venoso, no qual conflue tambem um ramo da grossa veia dorsal.

A differença entre as disposições dos dois lados consiste no facto em que á direita, a v. femural propriamente dita ressulta da fusão da grossa v. dorsal com a unica v. comitante da a. femural existente na porção distal do triangulo de Scarpa: á esquerda, a v. dorsal é maior e recebe como confluente um tronco venoso constituido, por sua vez, por duas vv. satellites da porção inferior da a. femural. Além disso, á direita, a grossa v. dorsal acompanha, na su: passagem da região posterior da coxa para a anterior, a 3.a a. perfurante: á esquerda, a grossa v. dorsal é, pelo contrario, satellite da 2.a perfurante da femural profunda.

Dissemos, mais acima, que as disposições que acabamos de descrever são bastante conhecidas pelos anatomistas e nem mesmo podem ser consideradas de extrema raridade.

Está tambem constatado que o comportamento das veias soffre um maior numero de desvios, consideraveis como variedades, que o das arterias e isso se verifica seja com as vv. superficiaes, independentes das arterias, seja com as vv. profundas que destas são satellites.

Variedades mesmo mais communs ou, pelo menos, mais evidentes são encontradas particularmente nas regiões de passagem entre os varios segmentos dos membros (cavo popiiteo, virîlhà, cotovello, axilla), ou do tronco, (collo) as quaes podem ser consideradas como verdadeiros confluentes venosos devido ás relações que se estabelecem, de preferencia nestas regiões entre as vv. superficiaes e as profundas, ao numero habitualmente consideravel de ramos venosos collateraes que se entrelaçam entre si e que 
acompanham os, rámos collateraes arteriosos correspondentes; e, finalmente porque as regiões de passagem, como CHARPY nota precisamente para o cavo popliteo, são justamente as partes nas quaes os movimentos de extensão ou de flexão, segundo tendem ou relaxam as fascias de revestimento, comprimem a rêde venosa ou, ao contrario, criam ao redor della uma athmosphera de fraca pressão que favorece o appello do sangue.

No que diz respeito ás disposições mais communs, e por isso consideradas como normaes, das vv profundas das regiões poplitéa e femural e ás suas relações geneticas com o comportamento das veias no nosso caso, é apenas necessario lembrar alguns factos sufficientes a esclarecerem de como resultaram os desvios de percurso dos troncos venosos, differentes á direita e á esquerda, no individuo que serviu para a nossa dissecção.

Primeiramente, a reunião tardia das vv. tibio-peroneiras, tibiaes anteriores, etc., póde dar origem a uma duplicidade do segmento distal da $\mathrm{v}$ poplitéa, considerada normal por ROBINEAU, como dissemos acima, e tambem lembrada por CHARPY. Nestes casos o tronco venoso popliteo constituir-se-ia sómente ao nivel da interlinha articular e o tronco unico seria reduzido a um comprimento de 5 a $7 \mathrm{cms}$.

A duplicidade, i. é, a existencia de dois troncos venosos na porção proximal $d_{u}$ cavo popliteo, mesmo quando se podem constatar anastomoses mais ou menos numerosas trocadas entre elles, pode apparecer como uma conservação dos dois troncos venosos principaes do segmento distal do mesmo cavo, devida a falta de união dos que constituem habitualmente a $\mathrm{v}$ poplitéa.

Quanrlo, somo nu nosso caso, das duas veias, ou melhor, dos dois grupos venosos longitudinaes occorrentes no segmento proximal do eavo popliteo, a veia mais calibrosa não acompanha a arteria axial no canal dos $\mathrm{mm}$. adductores, ainda que se faça ventral seguindo uma ou outra das aa. perfurantes da femural profunda, 6 bem possivel explicar o calibre preponderante da dita veia admittindo uma ectasia, provavelmente primitiva, de uma das vv. perfurantes e das anastomoses, aliás constantes, das vv. perfurantes entre si e da v. perf. inferior com a v. poplitéa.

Analogamente ao que acontece com as arterias, essas anastomoses, dorsaes ás inserções femuraes dos $\mathrm{mm}$. adductores, estabelecem uma via collateral de defluxo parallela á série de arcadas arteriosas, posta na região posterior da coxa; esta via collateral venosa não sómente une as vv poplitéas aos troncos venosos satellites da a. f. profunda, e isso independentemente das que acompanham a a. femural no canal de Hunter, mas, tambem ás vv. gluteas inferiores, vv. pudendas internas e porisso ao systema venoso directamente affluente da $v$. hypogastrica.

Quando uma das vv perfurantes e as relativas continuações anastomoticas dorsaes, aliás normaes, teem o valor de via principal de defluxo do sangue da região poplitéa, verifica-se, precisamente, as disposições por nós descriptas; queremos dizer, a continuação proximal da $\mathrm{v}$. poplitéa ou de 
um dos troncos principaes, resultantes de uma tardia ou incompleta união das vv. distaes, tem um percurso parcialmente independente da v. femural e da sua continuação como a. poplitéa; ainda o tronco venoso principal se conserva dorsal por uma extensão maior ou menor, segundo se faça ventral acompanhando a 3. perfurante (á direita no nosso caso), a 2." perfurante (á esquerda no nosso caso), a 1.: ou mesmo a a. circumflexa femuris medialis.

O tronco venoso principal póde ser mesmo completamente dorsal no seu inteiro percurso quando não acompanha os ramos collateraes da a. f. profunda, mas corre ao longo das anastomoses longitudinaes das aa. perfurantes e circumflexa f. medialis e o ramo descendente da a. glutea inferior: esta é a disposição que se verifica nos casos, aliás raros (BARCLAY, TESTUT dois casos), nos quaes a a. glutea inferior (a. ischiatica) representa o ramo principal de nutrição do membro inferior: esta disposição completamente dorsal da veia principal, póde porém, existir mesmo sendo a a. femural de calibre e em posição normaes; inversamente, a a. ischiatica póde ser o ramo principal do membro inferior, permanecendo as vv. poplitea e femural com a disposição habitual (MANNU).

Nas eventualidades acima consideradas, a porção proximal da a. poplitea e a porção da a. femural que vae desde o canal de Hunter até a origem da a. f. profunda, são acompanhadas por uma ou mais veias (e quando são mais, mesmo com disposição plexiforme) que representam no seu conjuncto uma via de defluxo secundaria: foi precisamente isto, com modalidades ligeiramente diversas, que verificamos no nosso caso.

A veia ou as veias que acompanham, de modo immediato, a porção proximal da a. poplitéa até o canal de Hunter, podem muito bem ser identificadas ás venas comitantes dos AA. (LANGER ,BRAUNE, CUNNINGHAM, AUVRAY, SPALTEHOLZ, CHIARUGI, PIERSOL e outros) e que CRUVEILHIER chama de collateraes: o mesmo valor de veias comitantes teem as que acompanham o tronco da a. femural, mesmo verificando-: e nellas o afluxo de veias dos grupos musculares (adductores, vasto medial) que estão em relação immediata com o feixe artero-venoso ventral. $\mathrm{g}$ calibre relativamente exiguo das veias comitantes da a. femural justifica a impressão de que estas sejam collateraes da $\mathrm{v}$ femural profunda, como acontece no nosso e em casos analogos. Têm-se então, nestas eventualidades, uma verdadeira inversão das disposições consideradas como normaes.

A explicação assím expressa do modo de origem da dispo :ição aqui tratada poupa-nos uma revista por demais minuciosa das chamadas anomalas ou variedades das veias em questão, as quaes estão consignadas em memorias especiaes ou nos tratados de anatomia que temos a nossa disposição.

De facto, na maioria dos classicos de Anatomia humana ou pelo menos nos que com as disposições que se consideram como normaes, porque são as mais frequentes, relatam tambem as menos constantes ou mesmo 
raras, considerando as como variedades ou anomalias (VELPEAU, THEILE, DUBRUEIL, CRUVEILHIEk, W KRAUSE em HENLE, QUAIN, CUNNINGHAM, ROMITI, CHARPY em POIRIER, TESTUT, SOBOTTA, RAUBER-KOPSCH, MORRIS, PIERSOL, CHIARUGI e outro:) como tambem em memorias especiaes (LANGER, BRAUNE, GRUBER GIACOMINI, HOUZE' PICQUE et PIGACHE, ROBINEAU, DAVIDSON, etc.), são feitas repetidas referencias a casos, mais ou menos parecidos ao que acabamos de illustrar. - Assim, é lembrada a duplicidade da $r$ poplitéa seja no segmento distal, seja em toda sua extensão (THEILE, GIACOMINI 3 casos, Testut, ChIARUGi, etc) - A duplicidade pode extender-se da $\mathrm{v}$ poplitéa á v. femural mesmo até a parte superior da coxa, e nestes casos as duas veias pódem ser exclusivamente satellites da a. poplitéa e da a. femural e anastomosadas entre si (TESTUT, CHARPY, etc.) - A v poplitéa normal ató á interlinha articular, divide-se superiormente em dois ramos; destes, um segue a arteria, o outro communica por uma larga anastomose, que parece uma bifurcação, com a v. femural profunda (GRUBER: na metade dos casos segundo HOUZE': lembrada algumas vezes por GIACOMINI). - A v. poplitéa, em vez de seguir a arteria no canal de Hunter, sóbe ao longo da região dorsal da coxa e, em alturas diversas, como no nosso caso, passa atravéz dos mm. adductores e se continua como v. f. profunda (VELPEAU, DUBRUEIL, CRUVEILHIER, KRAUSE, QUAIN, ELLIS, GRUBER, GIACOMINI etc): naturalmente não é excluida a existencia, nestes ultimos casos, embora não lembrada, de vv. comitantes ao segmento proximal da a. poplitéa e á a. femural (nosso caso): as vv. comitantes seriam, então, por certo muito exiguas de modo que a $v$ poplitéa tem a apparencia de se continuar exclusivamente com a v. f. profunda (GIACOMini, TEXTUT, Sperino, etc). - Ainda lembramos, novamente, a possivel continuação como via principal de defluxo da $\mathrm{v}$ poplitéa, na região posterior da coxa, até ás vv gluteas inferiores e por meio destas á v hypogastrica, concomitantemente ou não á uma a. ischiatica enormemente desenvolvidã.

São mesmo ben: conheciuas as lapellas (DUBRUEIL, GIACOMINI, TESTUT, CHARPY) ou a disposição plexiforme (BARKOW. AUVRAY) da $\mathrm{v}$ poplitéa, particularmente na parte media do cavo homonymo: estas u?timas disposiçõe podem ser verificadas mesmo quando a continuação da veia não é a commum, como nos casos de variações.

Deve-se particularmente notar que a continuação proximal da $v$ poplitea ná região corsal da coxá, seja como um "ramo de bifurcação" seja como um tronco que represente a via principal de defluxo, póde ser concomitante á feixes supranumerarios dos $\mathrm{mm}$. gemeos, particularmente lo m. gemeo interno, originarios do plano popliteo (GIACOMINI): natu ralmente, estes feixes accessorios dos $\mathrm{mm}$. gemeos, podem ser verificados mesmo sem a posição desviada das veias. 
O conhecimento li pos ihiltade de uma v. poplitea dupla, totalmente ou em parte do seu percurso, tem interesse para o cirurgião: na ligadura da a. poplitéa, na região lomonyma. aléh da união muito intima existente na bainha commum da veìa e da arteria, e da grande espessura da parede da veia, é de prever a possibilidade que têm os dois troncos venosos de apresentarem, com a arteria, relações de posição que pódem obstacular o isolamento desta. Er sentido inverso, a existencia de vv. comitantes mesmo pequenas, no segmento proximal da a. poplitea e na porção distal da a. femural, póde mascarar mais ou menos a arteria no canal de Hunter e na porç̃̃o inferior do trißngnlo de scarpa com a sua disposição plexiforme; esta disposição deve ser prevista pelos cirurgiões que be achariam em condições especiaes para isola: a arteria na occasião que devem proceder á sua ligadira $n$ s ditos pontos

Ainda, a lembrança da existencia de um grosso tronco dorsal ao femur, na parte media da coxa ou mesmo mais acima, deve estar presente na execução de uma amputação.

Em poucas palavras, o nosso caso, si bem que não seja novo nem raro, além do intere:se puramente descriptivo, póde ser lembrado naś applicações eventuaes da pratica cirurgica.

(Do Laboratoris de Anatomia Descriptiva da Faculdade de Medicina e Cirurgia de S. Paulo, dirigido pelo dr. A. Bovero.

S. Paulo, $9-\mathrm{VII}-925$.

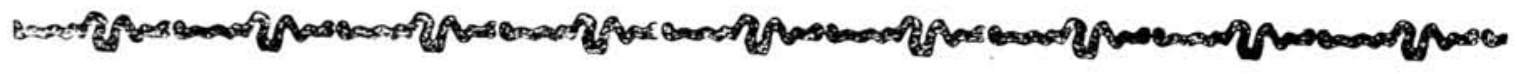

\section{LABORATORIO DE, MICRO SCOPIA}

E E

\section{ANALYSES CLINICAS}

D R

RLA DO CAIRMO N. 11 -

TELEPHONE GENTRAL $2 \dot{4} 63 \quad$ S. PAULO - 